\title{
circANRIL reduces vascular endothelial injury, oxidative stress and inflammation in rats with coronary atherosclerosis
}

\author{
PEIXIA SHI ${ }^{1}$, HONGLING JI ${ }^{2}$, HUAJUAN ZHANG ${ }^{3}$, JIAN YANG ${ }^{4}$, RUI GUO ${ }^{5}$ and JIANWEI WANG ${ }^{6}$ \\ ${ }^{1}$ No. 1 Department of Cardiology, The People's Hospital of Zhangqiu Area; ${ }^{2}$ Disinfection Supply Center and \\ ${ }^{3}$ Hospital-Acquired Infection Control Department, Jinan Zhangqiu District Hospital of Traditional Chinese Medicine; \\ Departments of ${ }^{4}$ Clinical Laboratory and ${ }^{5}$ Outpatients, The People's Hospital of Zhangqiu Area, Jinan, Shandong 250200; \\ ${ }^{6}$ ECG Room, W.F. Maternal and Child Health Hospital, Weicheng, Weifang 261000, P.R. China
}

Received February 7, 2020; Accepted June 5, 2020

DOI: $10.3892 /$ etm.2020.8956

\begin{abstract}
Effects of circular antisense non-coding RNA in the INK4 locus (circANRIL) on vascular endothelial injury, oxidative stress and inflammation in rats with coronary atherosclerosis were studied by establishing a rat model of coronary atherosclerosis in which circANRIL was differentially expressed. A total of 40 healthy Sprague Dawley (SD) rats were randomly divided into research group $(n=32)$ and control group $(n=8)$. In research group, a rat model of coronary atherosclerosis was established without special treatment. The blood calcium $\left(\mathrm{Ca}^{2+}\right)$ and lipid levels in the two groups were compared. After cell transfection, the rats were divided into blank group (untransfected), negative group (transfected with blank vector), circANRIL group (transfected with circANRIL overexpression plasmid) and circANRIL inhibitor group (transfected with circANRIL silencer). Then the levels of lactate dehydrogenase (LDH), superoxide dismutase (SOD), malondialdehyde (MDA), tumor necrosis factor- $\alpha$ (TNF- $\alpha$ ) and interleukin-6 (IL-6) in each group were compared. Western blotting was adopted to detect the expressions of phosphorylated p38 mitogen-activated protein kinase (p-p38MAPK), p38MAPK and glyceraldehyde 3-phosphate dehydrogenase (GAPDH). Finally, p-p38MAPK/GAPDH, p38MAPK/GAPDH and p-p38MAPK/p38MAPK were calculated. There were significant differences in the levels of serum $\mathrm{Ca}^{2+}$ and lipid between control group and research group $(\mathrm{P}<0.05)$. Besides, differences in LDH, SOD, MDA, TNF- $\alpha$ and IL- 6 in the supernatant in each group were statistically significant $(\mathrm{P}<0.05$ or $\mathrm{P}<0.01)$. Moreover, there were statistically significant differences in the gray values of
\end{abstract}

Correspondence to: Dr Jianwei Wang, ECG Room, W.F. Maternal and Child Health Hospital, 76 Qingnian Road, Weicheng, Weifang 261000, P.R. China

E-mail: mad17p@163.com

Key words: circANRIL, coronary artery disease, vascular endothelial injury, oxidative stress, inflammation
p-p38MAPK/GAPDH and p38MAPK/GAPDH and their ratio p-p38MAPK/p38MAPK in each group $(\mathrm{P}<0.05$ or $\mathrm{P}<0.01)$. Inhibiting the expression of circANRIL in coronary heart disease cases can reduce vascular endothelial injury, oxidative stress and inflammation.

\section{Introduction}

Coronary artery disease (CAD) is defined as an inflammatory disease developing mainly at branch points and bends of arteries, in which arterial blood flow continuously causes slight damage to the endothelial monolayer lining of the vessel wall. This process seems to be the key to the progression of atherosclerosis, because the maladaptation of endothelial cells (ECs) to this physiological disorder increases the susceptibility of branch points to pro-inflammatory states. Therefore, maladapted ECs make it easier for arterial branch points to circulate in the subendothelial space, lead to the inflow of oxidized low-density lipoproteins (ox-LDLs), and stimulate monocyte recruitment by releasing pro-inflammatory molecules (i.e., chemokines and adhesion molecules) (1). Subendothelial monocytes differentiate into macrophages, which locally proliferate and absorb ox-LDLs. Then the deposition of lipid into plaques leads to deterioration, thus triggering macrophages to become foam cells due to insufficient lipoprotein uptake. The progression of advanced lesions is featured with the accumulation of lipoproteins, apoptosis and necrosis of macrophage-derived foam cells, the formation of cholesterol crystals due to increased defective phagocytes, and the formation of cap smooth muscle cells (SMCs) (2). Hence, increased lesions in the advanced stage lead to severe reduction of arterial lumen and blood flow, decline of oxygen supply and rupture or dissolution of plaques, thus causing thrombosis. Analysis of the early development stage of atherosclerosis reveals that inflammation exerts a crucial effect in the development of atherosclerosis. Surprisingly, the emphasis of many current therapies applied to treat atherosclerosis and thrombosis has been put on coping with the onset of acute inflammatory states. It is noteworthy that statins can not only reduce the cholesterol level, but also play an anti-inflammatory role (3). This study, therefore, aims to more specifically reduce inflammatory activities during atherosclerosis using colchicine 
and interleukin (IL)-1 $\beta$ antibodies or other modes targeting IL-6 or IL-1 receptors (4).

A genome-wide association study (GWAS) and a single-cell sequencing database have revealed the existence of complex regulatory networks triggering cardiovascular diseases (5). In these networks, the heterogeneity and correlation of vascular cell population as well as their contributions to different stages of atherosclerosis are especially prominent. In addition, the GWAS has shown disease-related genetic variations in the non-protein coding sequence space, which are actively transcribed into non-coding ribonucleic acids (ncRNAs), i.e., micro RNAs (miRNAs) and long ncRNAs (lncRNAs) (6). These new-type ncRNAs exhibit differential expression in diseased tissues and function as epigenetic regulators for gene expression. Among them, the identification of molecules related to the interaction between phenotypes and cells and epigenetic regulation are new and selective therapeutic targets for the treatment of atherosclerosis.

Circular antisense non-coding RNA in the INK4 locus (circANRIL) is transcribed at the atherosclerotic CVD gene site on chromosome 9p21 (Chr9p21), which may have anti-atherosclerosis function. The high level of circANRIL expression in human vascular tissues is related to lower CAD severity. In terms of mechanism, circANRIL has been proven to bind to Pescadillo homolog 1 (PES1), an indispensable preassembly factor for $60 \mathrm{~S}$-ribosomes, thus possibly resulting in the damage of nucleic acid exonuclease-mediated pre-ribosomal RNA (rRNA) processing and ribosome biogenesis in VSMCs and macrophages (7). On the contrary, circANRIL induces p53 activation, leading to increased apoptosis, while the proliferation of vascular SMCs (VSMCs) and macrophages is decreased. Therefore, circANRIL might prevent atherosclerosis by inhibiting the excessive proliferation of cells in atherosclerotic plaques.

This research explored the effects of circANRIL on vascular endothelial injury, oxidative stress and inflammation in rats with coronary heart disease, and investigated a new method of treating CAD from the perspective of vascular biology.

\section{Materials and methods}

Materials. Sprague Dawley (SD) rats and feed were purchased from HFK Bioscience, circANRIL overexpression plasmids and silencers from JKJC Gene Technology Co., Ltd., rat IL-6 (cat. no. k4143-100) and TNF- $\alpha$ (cat. no. k1052-100) enzyme-linked immunosorbent assay (ELISA) kits from Jitai Yikai Biotechnology Co., Ltd., rabbit anti-rat p38 mitogen-activated protein kinase (p38MAPK, cat. no. ab170099) and phosphorylated (p)-p38MAPK antibodies (cat. no. ab47363) were from Abcam, rabbit anti-rat glyceraldehyde 3-phosphate dehydrogenase (GAPDH, cat. no. 10494-1-AP) from Proteintech, and factor VIII immunohistochemistry kit was from ZSGB-Bio Co., Ltd.

Modeling of coronary heart disease. A total of 40 healthy SD male rats aged 3-4 months, with an average body weight of $250.24 \pm 62.27 \mathrm{~g}$ were included and then, fed with normal diet and normal drinking water. At 7 days after feeding, the rats were randomly divided into research group $(n=32)$ and control group $(\mathrm{n}=8)$. The rats in control group received only basic feed and tap water with no special treatment, while those in research group were fed with high-fat diet. At the beginning of the experiment, vitamin D3 powder was injected into the right lower limb of rats at a dose of $3 \times 10^{6} \mathrm{U} / \mathrm{kg}$ every 30 days (8). The basic diet of the rats accounted for $94.30 \%$, and was supplemented with $2 \%$ cholesterol, $3 \%$ lard, $0.5 \%$ sodium cholate and $0.2 \%$ propylthiouracil. The experiment lasted three months. Then the rats were sacrificed and aortic specimens were collected. The rats received euthanasia via cervical dislocation (after being anesthetized using peritoneal administration of pentobarbital sodium at a dose of $40 \mathrm{mg} / \mathrm{kg}$ ). If there was calcification of the intima, the atherosclerosis model of the rat was successful. The success rate of the model was $70 \%$, and the death rate was $10 \%$. The cause of death may be related to the loss of appetite, poor resistance and diarrhea. The study was approved by the Ethics Committee of the People's Hospital of Zhangqiu Area (Jinan, China).

Detection of blood calcium $\left(\mathrm{Ca}^{2+}\right)$ and lipid. The rats were anesthetized using pentobarbital sodium at a dose of $40 \mathrm{mg} / \mathrm{kg}$ intraperitoneally. Blood $(2 \mathrm{ml})$ was collected from the abdominal aorta of rats. After centrifugation at $4^{\circ} \mathrm{C}, 10,500 \mathrm{x} \mathrm{g}$ for $5 \mathrm{~min}$, plasma was separated, and $1 \mathrm{ml}$ of blood $\mathrm{Ca}^{2+}$ and $1 \mathrm{ml}$ of lipid were measured. The expression of total cholesterol (TC), triglyceride (TG), high-density lipoprotein cholesterol (HDLC), low-density lipoprotein cholesterol (LDLC) and blood $\mathrm{Ca}^{2+}$ was detected using a biochemical analyzer.

Cell culture method and verification of cell purity. In a sterile environment, the proximal coronary artery of the rats was taken out and washed in phosphate buffer containing double antibodies (1\% penicillin and $1 \%$ streptomycin). After centrifugation at $4^{\circ} \mathrm{C}, 300 \mathrm{x}$ g for $10 \mathrm{~min}$, the precipitation was collected and cultured in $20 \%$ fetal bovine serum (FBS), Dulbecco's modified Eagle's medium-F12 (DEME-F12) (Gibco) in an environment with moderate humidity and $5 \% \mathrm{CO}_{2}$ at $37^{\circ} \mathrm{C}$. It was observed that ECs crawled out from the edge of the tissue mass and gradually extended outward within $\sim 7$ days. At this time, with the tissue mass removed, the cells were washed 3 times with phosphate-buffered saline (PBS) to remove the residual culture medium. Then $0.25 \%$ trypsin was added to the culture dish for digestion. When the cells contracted and became round ( $\sim 10 \mathrm{~min}), 5 \mathrm{ml}$ of $20 \%$ FBS DEME-F12 medium was added, and the cells were inoculated into a $25 \mathrm{~cm}^{2}$ culture flask. Subsequently, $2 \mathrm{ml}$ of cells were inoculated into a 6-well plate (with pre-sterilized glass plates) at a density of $1.2 \times 10^{5}$ cells $/ \mathrm{ml}$. When the cells reached $70 \%$ fusion rate, the detection started. ECs were flat and short fusiform or polygon, and they contained only factor VIII. Through immunochemical staining as well as the specific binding of antibodies and antigens, the nucleus containing factor VIII was yellow, while that without factor VIII was dark blue, which could be used to distinguish cells.

Cell transfection method. One day before transfection, the transfected cells were seeded into a 12 -well plate at a density of $2 \times 10^{5} / \mathrm{cm}^{2}$. Transfection was performed using Lipofectamine $^{\text {TM }} 2000$ (Invitrogen; Thermo Fisher Scientific, Inc.). When the fusion rate reached $\sim 70 \%$, circANRIL 
Table I. Comparison of the levels of blood $\mathrm{Ca}^{2+}$ and lipid in rats (mean $\pm \mathrm{SD}, \mathrm{mmol} / \mathrm{l}$ ).

\begin{tabular}{lrccccc}
\hline Group & $\mathrm{n}$ & $\mathrm{TC}$ & $\mathrm{TG}$ & HDLC & LDLC & $\mathrm{Ca}^{2+}$ \\
\hline Control group & 8 & $5.12 \pm 0.75$ & $4.52 \pm 0.86$ & $2.63 \pm 1.17$ & $2.03 \pm 0.93$ & $3.67 \pm 0.14$ \\
Research group & 32 & $16.73 \pm 1.72^{\mathrm{a}}$ & $1.19 \pm 0.04^{\mathrm{b}}$ & $2.01 \pm 0.94^{\mathrm{b}}$ & $7.83 \pm 1.39^{\mathrm{b}}$ & $2.42 \pm 0.07^{\mathrm{b}}$ \\
\hline
\end{tabular}

${ }^{\mathrm{a}} \mathrm{P}<0.01$ and ${ }^{\mathrm{b}} \mathrm{P}<0.05$ vs. control group. TC, total cholesterol; TG, triglyceride; HDLC, high-density lipoprotein cholesterol; LDLC, low-density lipoprotein cholesterol.

transfection was carried out according to the instructions of the transfection kit, and corresponding negative control experiments were performed to form blank group (untransfected cells), negative group (transfected with blank vector), circANRIL group (transfected with circANRIL overexpression plasmid, $20 \mu \mathrm{M}$ ), and circANRIL inhibitor group (transfected with circANRIL silencer siRNA, $20 \mu \mathrm{M}$ ). After $18 \mathrm{~h}$ of transfection, the fluorescent tag was added to detect the transfection efficiency. Following incubation for $24 \mathrm{~h}$, subsequent experimental steps were conducted.

Lactate dehydrogenase ( $\mathrm{LDH}$ ) detection. When cells were damaged, LDH was released into the supernatant. Since LDH in the supernatant did not easily react with other components, the amount of LDH in the supernatant could be measured to reflect the number of dead and damaged cells. Hundred milliliters of the supernatant was absorbed by each well of a 96-well plate. Then the supernatant was aspirated carefully to prevent cells from aspiration. Thereafter, $100 \mathrm{ml}$ of working solution was added to each well, and the well was coated with aluminum foil to avoid light. After reacting at room temperature for $30 \mathrm{~min}, 50 \mathrm{ml}$ of termination solution was added to each well, and the absorption amount at $490 \mathrm{~nm}$ was immediately measured using a microplate reader. Finally, the cell damage rate was calculated according to the following formula: Cell damage rate $=$ sample well/high control well $\times 100 \%$.

Determination of superoxide dismutase (SOD) and malondialdehyde (MDA). The supernatant in each group was collected, and the content of SOD in rat serum was determined by xanthine oxidase method. After that, free radicals produced by reactions of xanthine and xanthine oxidase were detected. Next, the content of MDA in serum was determined by thiobarbituric acid colorimetry according to the instructions of a kit provided by Shanghai Jinghua Instruments Co., Ltd.

Detection of IL- 6 and TNF- $\alpha$ by ELISA. Cell supernatant was collected, and protein concentration was determined by bicinchoninic acid assay (BCA) (Pierce; Thermo Fisher Scientific, Inc.). According to the instructions of the ELISA kit, the micropores of rat IL- $1 \beta$, IL- 6 and TNF- $\alpha$ capture antibodies were pre-coated, and then samples, standard substances and horseradish peroxidase (HRP)-labeled antibodies were sequentially added and thoroughly washed. When the substrate TMB was used for color development, TMB turned blue under the catalysis of peroxidase and yellow under the action of acid. Its color was positively correlated with the content of IL-6 and TNF- $\alpha$ in the supernatant of rat ECs. The optical density was measured at $450 \mathrm{~nm}$ using the microplate reader, and the concentrations of IL- 6 and TNF- $\alpha$ were calculated.

Detection of the protein expression of $p$-p38MAPK and p38MAPK in the supernatant via western blotting. Cell supernatant was collected from each group. Based on the instructions of the BCA kit, the protein concentration in ECs was detected. Then, 5X sample buffer was added and boiled, and proteins $(10 \mu \mathrm{g})$ were separated by polyacrylamide gel electrophoresis and transferred onto a polyvinylidene fluoride membrane. Next, the proteins were sealed with $10 \%$ skim milk, blocked with $2 \%$ phosphoenzyme inhibitor p-Smad3, and incubated with anti-p-p38MAPK antibody $(1: 2,000)$ and p38MAPK antibody $(1: 1,000)$ after washing, followed by incubation overnight at room temperature of $4{ }^{\circ} \mathrm{C}$. Then the membranes were incubated with goat horseradish peroxidase-conjugated goat anti-rabbit IgG H\&L secondary antibody (1:1,000; cat. no. ab7090; Abcam) at $25^{\circ} \mathrm{C}$ for $1 \mathrm{~h}$. Thereafter, the exposure was performed by Bio-Rad gel imaging system, and Image Lab 6.0 software was adopted for processing. The gray value was calculated, and the ratio of the gray value of the target band to the corresponding internal reference GAPDH reflected the relative expressions of p-p38MAPK and p38MAPK.

Statistical analysis. Data in each group were collected using Statistical Product and Service Solutions (SPSS) 19.0 software (SPSS Inc.) and analyzed as (mean \pm SD). Differences between two groups were analyzed by using the Mann Whitney U test. Comparison between multiple groups was done using the Kruskal-Wallis test with Dunn's post hoc test.

\section{Results}

Comparison of the levels of blood $\mathrm{Ca}^{2+}$ and lipid in rats. Research group had higher levels of TC, TG and LDLC $(\mathrm{P}<0.05$ or $\mathrm{P}<0.01)$, and lower levels of HDLC and $\mathrm{Ca}^{2+}$ than control group $(\mathrm{P}<0.05)$ (Table I).

EC identification results. Within one week, cell adhesion was decreased and growth became slow. After 7 days, a large number of cells grew out of the clump in good growth conditions. Under an inverted microscope, the cells adhered to the wall and grew in short spindle or polygonal shape (Fig. 1A). Factor VIII is released from ECs. Based on this principle, factor VIII was detected by immunohistochemistry. As the basis for identifying ECs, brown paving-like cells were ECs (Fig. 1B). 

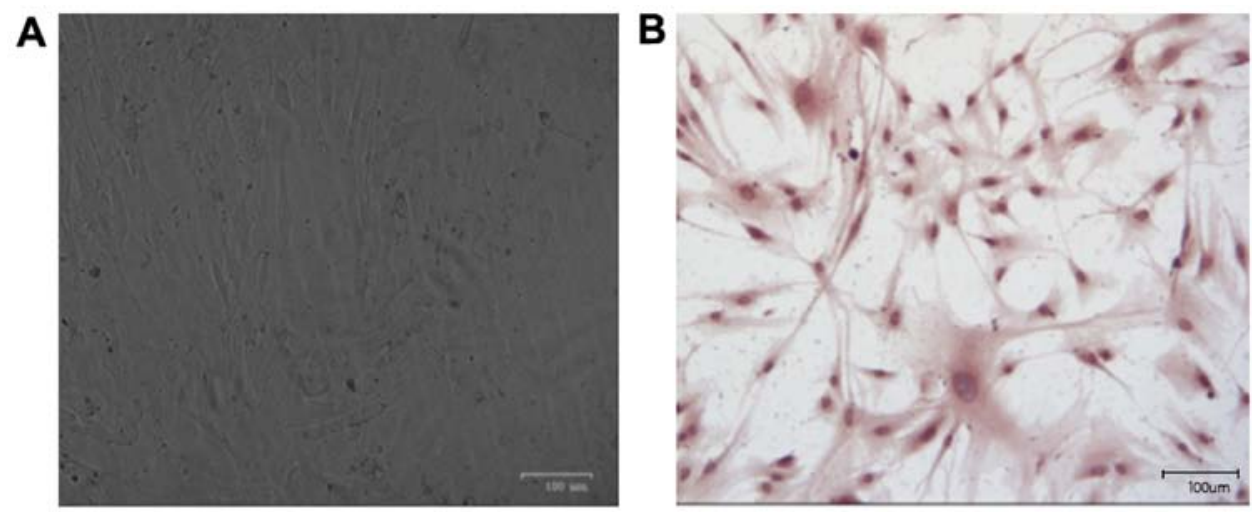

Figure 1. (A) EC morphology observed under an inverted microscope. (magnification, x100). (B) Fluorescence staining results of EC factor VIII using immunohistochemistry (magnification, x200). EC, endothelial cell.

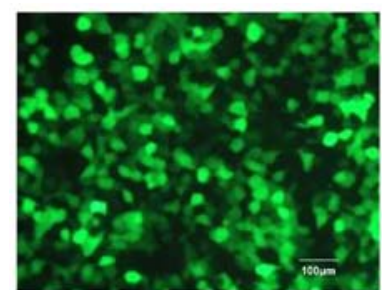

Negative

group

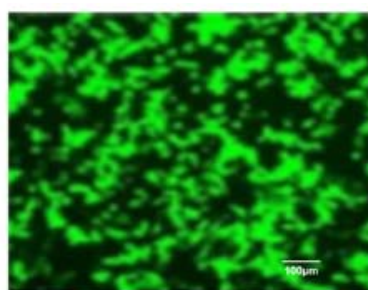

CircANRIL

group

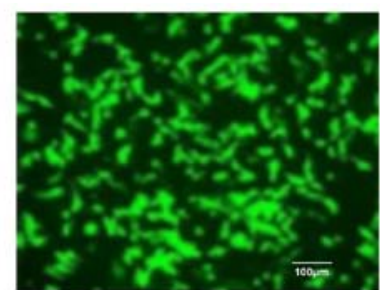

CircANRIL inhibitor group

Figure 2. Transfection efficiency of each group of cells (magnification, x200). circANRIL, circular antisense non-coding RNA in the INK4 locus.

A

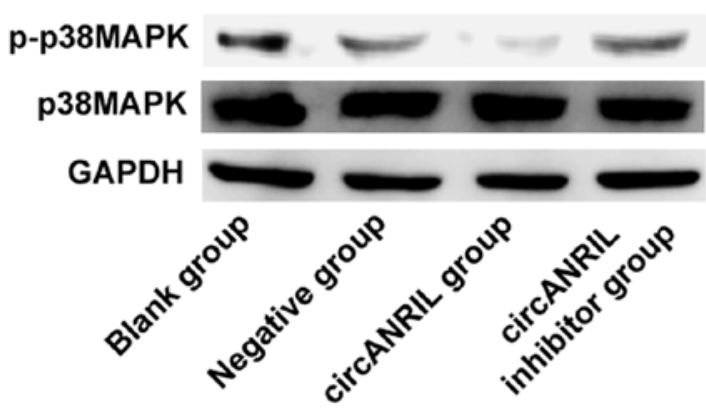

B

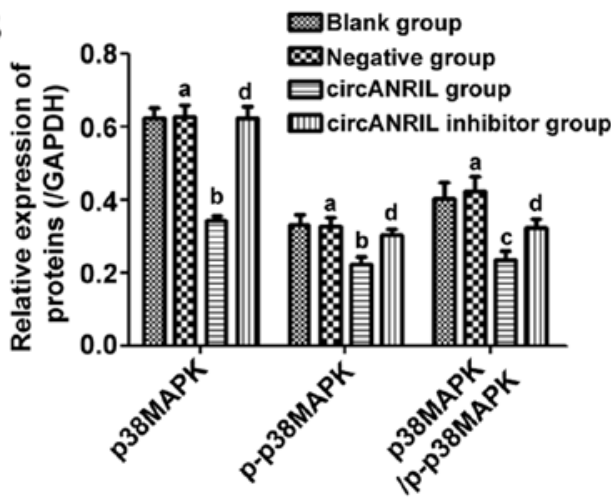

Figure 3. (A) Expression of p-p38MAPK, p38MAPK and GAPDH proteins. (B) Ratio of gray value of each target protein to GAPDH. ${ }^{a} \mathrm{P}>0.05$ vs. blank group; ${ }^{\mathrm{b}} \mathrm{P}<0.05,{ }^{\mathrm{C}} \mathrm{P}<0.01$ and ${ }^{\mathrm{d}} \mathrm{P}>0.05$ vs. negative group. p38MAPK, p38 mitogen-activated protein kinase; GAPDH, glyceraldehyde 3-phosphate dehydrogenase; circANRIL, circular antisense non-coding RNA in the INK4 locus.

Cell transfection results. Negative control (empty vector), circANRIL overexpression plasmid and cANRIL silencer were transfected into ECs, separately. The transfection efficiency was $>70 \%$ (Fig. 2), which met the requirements for the next experiment.

Determination of EC damage rate in each group. Compared with that in blank group, the damage rate in negative group did not change significantly $(\mathrm{P}>0.05)$. Besides, in comparison with that in the negative group, the cell damage rate in the circANRIL group was decreased $(\mathrm{P}<0.01)$, but it did not change in circANRIL inhibitor group ( $\mathrm{P}>0.05)$ (Table II).
Expression levels of SOD and MDA in ECs in each group. Compared with those in blank group, there were no significant changes in SOD and MDA releases in negative group $(\mathrm{P}>0.05)$. Compared with those in negative group, SOD and MDA releases in circANRIL group were remarkably decreased $(\mathrm{P}<0.01, \mathrm{P}<0.05)$. In addition, no obvious changes in SOD and MDA releases were found between negative group and circANRIL inhibitor group ( $\mathrm{P}>0.05)$ (Table III).

Expression levels of IL-6 and TNF- $\alpha$ in ECs in each group. Compared with those in blank group, there were no evident changes in IL-6 and TNF- $\alpha$ releases in negative group 
Table II. Comparison of EC damage rate in each group (mean $\pm \mathrm{SD}, \%)$.

\begin{tabular}{lll}
\hline Group & $\mathrm{n}$ & Damage rate \\
\hline Blank group & 6 & $38.67 \pm 4.78$ \\
Negative group & 6 & $40.62 \pm 4.65^{\mathrm{a}}$ \\
circANRIL group & 6 & $21.76 \pm 3.58^{\mathrm{b}}$ \\
circANRIL inhibitor group & 6 & $39.72 \pm 4.97^{\mathrm{c}}$
\end{tabular}

${ }^{a} \mathrm{P}>0.05$ vs. blank group; ${ }^{b} \mathrm{P}<0.01$ and ${ }^{\mathrm{C}} \mathrm{P}>0.05$ vs. negative group. EC, endothelial cell; circANRIL, circular antisense non-coding RNA in the INK4 locus.

Table III. SOD and MDA in ECs in each group (mean $\pm \mathrm{SD}, \mathrm{n}=8$ ).

\begin{tabular}{lll}
\hline Group & SOD (U/ml) & MDA (mmol/l) \\
\hline Blank group & $50.67 \pm 7.78$ & $47.25 \pm 9.27$ \\
Negative group & $52.67 \pm 8.03^{\mathrm{a}}$ & $46.85 \pm 8.75^{\mathrm{a}}$ \\
circANRIL group & $89.52 \pm 12.38^{\mathrm{b}}$ & $21.76 \pm 3.58^{\mathrm{c}}$ \\
circANRIL inhibitor group & $53.62 \pm 11.17^{\mathrm{d}}$ & $45.82 \pm 8.67^{\mathrm{d}}$
\end{tabular}

${ }^{\mathrm{a}} \mathrm{P}>0.05$ vs. blank group; ${ }^{\mathrm{b}} \mathrm{P}<0.01,{ }^{\mathrm{c}} \mathrm{P}<0.05$ and ${ }^{\mathrm{d}} \mathrm{P}>0.05$ vs. negative group. SOD, superoxide dismutase; MDA, malondialdehyde; EC, endothelial cell; circANRIL, circular antisense non-coding RNA in the INK4 locus.

( $\mathrm{P}>0.05)$. Compared with those in negative group, IL-6 and TNF- $\alpha$ releases in circANRIL group were reduced $(\mathrm{P}<0.05)$, but they did not change in circANRIL inhibitor group (P>0.05) (Table IV).

Expression of p-p38MAPK and p38MAPK proteins in ECs. p-p38MAPK, p38MAPK and p38MAPK/p-p38MAP did not markedly change in blank group compared with those in negative group $(\mathrm{P}>0.05)$. In comparison with those in negative group, the expression of p-p38MAPK and p38MAPK were decreased $(\mathrm{P}<0.05)$, and $\mathrm{p} 38 \mathrm{MAPK} / \mathrm{p}-\mathrm{p} 38 \mathrm{MAP}$ distinctly declined in circANRIL group $(\mathrm{P}<0.01)$, but they showed no changes in circANRIL inhibitor group ( $\mathrm{P}>0.05)$ (Fig. 3).

\section{Discussion}

The progressive lesions of human coronary atherosclerosis can be reversed by calcium antagonists, while the traditional high-fat diet fed animals have only cholesterol deposition, but no obvious calcium deposition. It has been reported that high dose of vitamin D3 can induce arterial wall calcification, which can be reversed by calcium antagonists. Therefore, rat model of coronary atherosclerosis induced by high-fat diet combined with vitamin D3 conforms to human coronary atherosclerosis better. In this study, high-fat diet combined with vitamin D3 were used for modeling rat coronary atherosclerosis.

In the study of cardiovascular diseases, it is urgent to find new methods for the diagnosis, treatment and prognosis of
Table IV. Expression levels of IL-6 and TNF- $\alpha$ in ECs in each group (mean $\pm \mathrm{SD}, \mathrm{n}=8$ ).

\begin{tabular}{lcc}
\hline Group & IL-6 (pg/ml) & TNF- $\alpha(\mathrm{pg} / \mathrm{ml})$ \\
\hline Blank group & $100.87 \pm 10.78$ & $47.83 \pm 5.62$ \\
Negative group & $99.87 \pm 10.36^{\mathrm{a}}$ & $44.72 \pm 5.03^{\mathrm{a}}$ \\
circANRIL group & $67.92 \pm 6.84^{\mathrm{b}}$ & $26.61 \pm 3.83^{\mathrm{b}}$ \\
circANRIL inhibitor group & $107.56 \pm 11.62^{\mathrm{c}}$ & $45.62 \pm 5.93^{\mathrm{c}}$ \\
\hline
\end{tabular}

${ }^{\mathrm{a}} \mathrm{P}>0.05$ vs. blank group; ${ }^{\mathrm{b}} \mathrm{P}<0.05$ and ${ }^{\mathrm{c}} \mathrm{P}>0.05$ vs. negative group. IL-6, interleukin-6; TNF- $\alpha$, tumor necrosis factor- $\alpha$; EC, endothelial cell; circANRIL, circular antisense non-coding RNA in the INK4 locus.

coronary heart disease. At present, coronary heart disease is still the major cause of death worldwide. People are very interested in the discovery of molecular biomarkers, which can supplement the traditional cardiovascular risk score in clinical decision-making and help to stratify patients for personalized treatment. With the development of next-generation sequencing and bioinformatics techniques, the interest in circRNAs has gradually emerged in recent years. It is noteworthy that molecules considered as 'junk RNAs' for 20 years are now one of the most attractive molecules (9). CircRNAs exert crucial effects in physiological and pathological processes. They are present not only in different tissues but also in body fluids, such as blood, saliva and semen, confirming its potential role as clinical biomarkers (10).

CircRNAs are produced by exon or intron sequences and conserved among species, and they exhibit specific expression in tissues. ANRIL is expressed in ECs, VSMCs, inflammatory cells and tissues affected by atherosclerosis. It has been proved that silencing ANRIL in human aortic VSMCs by siRNAs selectively targeting exon 1 or exon 19 can differentially regulate apoptosis, proliferation, inflammation and ECM remodeling, i.e. BCL2A1, baculoviral IAP repeat-containing protein 3 , cadherin 5 and heparin binding EGF-like growth factor, show isotype-specific regulatory characteristics (11). Lo Sardo et al (12) recently induced pluripotent stem cell-derived VSMCs from CAD risk and non-risk individuals using TALEN technique, and deleted regions corresponding to $60 \mathrm{~kb}$ risk haplotypes (depleted coding genes). Transcription profile analysis demonstrated that individual CAD risk VSMC displays altered gene expression patterns, similar to previous findings in CAD risk individuals. Besides, the individual VSMC also shows abnormal adhesion, contraction and proliferation. Deleting risk haplotypes can save the normal phenotype of VSMCs. On the contrary, risk phenotypes induced by lncRNA-ANRIL in non-risk VSMCs are forced to be expressed, have no degradation mediated by nucleic acid exonucleases and are more stable than most linear RNAs (13) as circRNAs form a covalently closed continuous loop, which is a great advantage in clinic. CircRNAs modulate gene expression through various mechanisms. In fact, circRNAs can act as miRNA sponges and play a competitive role in binding miRNAs through post-transcriptional regulation (14). Additionally, circRNAs can also regulate transcription by interacting with 
nuclear small RNAs or RNA polymerase II (15). Moreover, circRNAs are able to regulate RNA splicing by binding to transcription factors (16). The value of circRNAs in the diagnosis and prediction of heart diseases needs exploration. Emerging clinical and experimental studies verify that circRNA may be potential key regulatory factors for the onset and development of CAD. Burd et al (17) found that the circANRIL expression is related to INK4/ARF transcription and atherosclerotic disease risk. It is interesting that the genetic variation of $\mathrm{Chr} 9 \mathrm{p} 21$ is the most widely recognized CAD genetic risk, which can regulate ANRIL splicing and circANRIL production (18). circANRIL can protect atherosclerosis by controlling the maturation of ribosomal RNAs and regulating the formation pathway of atherosclerosis (19). Specifically, circANRIL binds to PES1, which is an essential 60S-ribosome preassembly factor, thus impairing nucleic acid exonuclease-mediated pro-rRNA processing and ribosome biogenesis in VSMCs and macrophages. Therefore, circANRIL induces nucleolar stress and p53 activation and causes induction of apoptosis and inhibition of proliferation, which is the key to atherosclerosis (20). Besides, reducing the expression of circANRIL can prevent coronary atherosclerosis by decreasing apoptosis of vascular ECs and the expression of inflammatory factors (20). In the study of Song et al (21) the expression levels of TC, TG, LDL, IL-1, IL-6, matrix metalloproteinase 9 and C-reactive protein in circANRIL group are low, and Bax, caspase-3 and the apoptosis rate of ECs are reduced, while the expression levels of HDL and Bcl-2 mRNAs and proteins are increased. Conversely, the changes in the expression levels in circANRIL overexpression group are the opposite to those in circANRIL low-expression group. The results confirm the protective effect of circANRIL in atherosclerosis, but when the dose exceeds a certain threshold, the protective effect will resume.

Holdt et al (7) demonstrated that circANRIL is involved in the maturation of VSMC and macrophage rRNAs. Specifically, pre-rRNA treatment and ribosome biosynthesis are impaired, resulting in nucleolar pressure, activation of p53, and subsequently increased cell apoptosis and reduced proliferation rate by binding circANRIL to PES1. Therefore, the protective effect of atherosclerosis on the formation of atherosclerotic plaques is proposed, which involves inhibiting cell proliferation in the early stage of atherosclerotic plaque development. This indicates that the genotype of Chr9p21 is essential for modulating the balance between linearity and circANRIL levels in VSMCs and macrophages. Therefore, changes in the percentage of ANRIL linear isotypes will be beneficial to atherosclerosis. In fact, the expression of exogenous circANRIL has been proven to be beneficial in the rat model of coronary atherosclerosis (21).

In conclusion, inhibition of circANRIL expression in coronary heart disease can reduce vascular endothelial injury, oxidative stress and inflammatory responses, thus providing new ideas and molecular biological methods for the diagnosis and treatment of CAD.

\section{Acknowledgements}

Not applicable.

\section{Funding}

No funding was received.

\section{Availability of data and materials}

All data generated or analyzed during this study are included in this published article.

\section{Authors' contributions}

PS and JW designed the study and performed the experiments. PS and HJ established the animal models. HZ was also involved in the conception of the study. JY and RG analyzed the data. PS and JW wrote the manuscript. All authors read and approved the final manuscript.

\section{Ethics approval and consent to participate}

The study was approved by the Ethics Committee of the People's Hospital of Zhangqiu Area (Jinan, China).

\section{Patient consent for publication}

Not applicable.

\section{Competing interests}

The authors declare that they have no competing interests.

\section{References}

1. Li GM, Zhang CL, Rui RP, Sun B and Guo W: Bioinformatics analysis of common differential genes of coronary artery disease and ischemic cardiomyopathy. Eur Rev Med Pharmacol Sci 22: 3553-3569, 2018

2. Natarelli L and Weber C: Next-generation therapeutic concepts for atherosclerosis: Focus on cell specificity and noncoding RNAs. Thromb Haemost 119: 1199-1201, 2019.

3. Martini E, Stirparo GG and Kallikourdis M: Immunotherapy for cardiovascular disease. J Leukoc Biol 103: 493-500, 2018.

4. Busygina K, Denzinger V, Bernlochner I, Weber C, Lorenz R and Siess W: Btk Inhibitors as first oral atherothrombosis-selective antiplatelet drugs? Thromb Haemost 119: 1212-1221, 2019.

5. Conn SJ, Pillman KA, Toubia J, Conn VM, Salmanidis M, Phillips CA, Roslan S, Schreiber AW, Gregory PA and Goodall GJ: The RNA binding protein quaking regulates formation of circRNAs. Cell 160: 1125-1134, 2015.

6. Ivanov A, Memczak S, Wyler E, Torti F, Porath HT, Orejuela MR, Piechotta M, Levanon EY, Landthaler M, Dieterich C, et al: Analysis of intron sequences reveals hallmarks of circular RNA biogenesis in animals. Cell Rep 10: 170-177, 2015.

7. Holdt LM, Stahringer A, Sass K, Pichler G, Kulak NA, Wilfert W, Kohlmaier A, Herbst A, Northoff BH, Nicolaou A, et al: Circular non-coding RNA ANRIL modulates ribosomal RNA maturation and atherosclerosis in humans. Nat Commun 7: 12429, 2016.

8. Gou SH, Liu BJ, Han XF, Wang L, Zhong C, Liang S, Liu H, Qiang Y, Zhang Y and Ni JM: Anti-atherosclerotic effect of Fermentum rubrum and Gynostemma pentaphyllum mixture in high-fat emulsion- and vitamin D3-induced atherosclerotic rats. J Chin Med Assoc 81: 398-408, 2018.

9. Qu S, Zhong Y, Shang R, Zhang X, Song W, Kjems J and Li H: The emerging landscape of circular RNA in life processes. RNA Biol 14: 992-999, 2017.

10. Salzman J, Gawad C, Wang PL, Lacayo N and Brown PO: Circular RNAs are the predominant transcript isoform from hundreds of human genes in diverse cell types. PLoS One 7: e30733, 2012. 
11. Congrains A, Kamide K, Katsuya T, Yasuda O, Oguro R, Yamamoto K, Ohishi $M$ and Rakugi H: CVD-associated non-coding RNA, ANRIL, modulates expression of atherogenic pathways in VSMC. Biochem Biophys Res Commun 419: 612-616, 2012.

12. Lo Sardo V, Chubukov P, Ferguson W, Kumar A, Teng EL, Duran M, Zhang L, Cost G, Engler AJ, Urnov F, et al: Unveiling the role of the most impactful cardiovascular risk locus through haplotype editing. Cell 175: 1796-1810.e20, 2018.

13. Jeck WR, Sorrentino JA, Wang K, Slevin MK, Burd CE, Liu J, Marzluff WF and Sharpless NE: Circular RNAs are abundant, conserved, and associated with ALU repeats. RNA 19: 141-157, 2013.

14. Hansen TB, Jensen TI, Clausen BH, Bramsen JB, Finsen B, Damgaard CK and Kjems J: Natural RNA circles function as efficient microRNA sponges. Nature 495: 384-388, 2013.

15. Han YN, Xia SQ, Zhang YY, Zheng JH and Li W: Circular RNAs: A novel type of biomarker and genetic tools in cancer. Oncotarget 8: 64551-64563, 2017.

16. Zeng X, Lin W, Guo M and Zou Q: A comprehensive overview and evaluation of circular RNA detection tools. PLOS Comput Biol 13: e1005420, 2017.

17. Burd CE, Jeck WR, Liu Y, Sanoff HK, Wang Z and Sharpless NE: Expression of linear and novel circular forms of an INK4/ARF-associated non-coding RNA correlates with atherosclerosis risk. PLoS Genet 6: e1001233, 2010.
18. Li CY, Ma L and Yu B: Circular RNA hsa circ 0003575 regulates oxLDL induced vascular endothelial cells proliferation and angiogenesis. Biomed Pharmacother 95: 1514-1519, 2017.

19. Geng HH, Li R, Su YM, Xiao J, Pan M, Cai XX and Ji XP: The circular RNA Cdrlas promotes myocardial infarction by mediating the regulation of miR-7a on its target genes expression. PLoS One 11: e0151753, 2016.

20. Zhao Z, Li X, Gao C, Jian D, Hao P, Rao L and Li M: Peripheral blood circular RNA hsa_circ_0124644 can be used as a diagnostic biomarker of coronary artery disease. Sci Rep 7: 39918, 2017.

21. Song CL, Wang JP, Xue X, Liu N, Zhang XH, Zhao Z, Liu JG, Zhang CP, Piao ZH, Liu Y, et al: Effect of circular ANRIL on the inflammatory response of vascular endothelial cells in a rat model of coronary atherosclerosis. Cell Physiol Biochem 42: 1202-1212, 2017.

This work is licensed under a Creative Commons Attribution-NonCommercial-NoDerivatives 4.0 International (CC BY-NC-ND 4.0) License. 